\title{
Endocardial Fibroelastosis
}

National Cancer Institute

\section{Source}

National Cancer Institute. Endocardial Fibroelastosis. NCI Thesaurus. Code C98922.

A rare disorder characterized by diffuse thickening of the endocardium. It presents with unexplained heart failure. 